Zusammenhang zwischen Angst und politischer Apathie", ausführliche Antworten des Verfassers auf die Fragen "Gibt es einen Aggressionstrieb?" und "Wie kommen wir zu einer 'konstitutionellen Intoleranz' gegen den Krieg?" folgen. In den weiteren Beiträgen holt Hom zunächst philosophiegeschichtlich weiter aus, stellt die konträren Aussagen von Rousseau und Hobbes gegenüber; außerdem werden von ihm Forschungsergebnisse aus den Fachbereichen Psychologie, Soziologie, Anthropologie und Ethologie referiert, teils mit lesenswerten, kritischen Anmerkungen versehen. Schließlich wendet sich Horn entschieden gegen monokausale Erklärungen und fordert programmatisch: "Gewalt- und Aggressionsanalysen sind letzten Endes nur als gesamtgesellschaftliche Analysen denkbar, in denen die jeweiligen Funktionszusammenhänge ... sowohl mit ihrem Eigengewicht, aber eben nur mit ihrem relativen Eigengewicht, im Gesamtkontext zu berücksichtigen sind" (S. 85).

In weiteren Texten geht Hom auf Kriegsursachen und -angst ein, versucht mit Hilfe eines "multifaktoriellen" Ansatzes aggressives Verhalten von bestimmten Personen oder Gruppen genauer zu ergründen - etwa die 1968 verübten Morde des amerikanischen Leutnants Calley im vietnamesischen Dorf MyLai oder bei dem zeitgenössischen Rechtsextremismus hierzulande. Insgesamt wird bei diesem Buch deutlich, daß es dem Autor immer um die Frage geht, wie Aggression und Gewalt durch uneingelöste, im gesellschaftlichen Alltag nicht anerkannte persönliche Bedürfnisse und Wünsche produziert werden, aber auch, wie sie durch pychologische Selbstaufklärung und durch politische Lemprozesse überwunden werden können. Ein Buch, das hoffen läßt.

Peter Barth

\title{
Armed Separatism in Southeast Asia
}

Edited by Lim Joo-Jock and Vani S. Singapore (RSSP/ISEAS) 1984, 270 pp.

Der von den zahlreichen Opfern und Kritikern des letzten Indochina-Krieges antizipierte "Sieg im Volkskrieg" werde, so die allgemeine Hoffnung, den Menschen dauerhaften Frieden und eine gedeihliche Entwicklung zum Besseren - sprich: in sozialistischen Verhältnissen - bescheren. Doch einmal mehr zeigte sich die List der Geschichte von ihrer unbarmherzigsten Seite; von diesen Idealvorstellungen ist Indochina - vielleicht mit Ausnahme von Laos - mindestens so weit entfernt wie Mitte der 70er Jahre. Die wirtschaftlichen Schwierigkeiten des mittlerweile wiedervereinigten Vietnam, sein - vorsichtig formuliert "getrübtes" Verhältnis zum Nachbarn Kampuchea und die relativierte "ewige Freundschaft und Waffenbrüderschaft" mit der VR China liefer(te)n noch immer am "Vietnam-Syndrom" laborierenden politischen Kräften in den USA (nicht zuletzt ihrer Filmindustrie) unverhoffte Legitimationsversuche. 
Ähnlich wurde und wird im Falle der Philippinen verfahren - dem nach Vietnam herausragenden (Medien)Ereignis. Langjährig war eine sich gegenüber ausländischen Interessen funktional verhaltene Diktatur gestützt worden (unvergessen George Bushs Laudatio auf Marcos, in der er diesem anläßlich der Proklamation der "Neuen Republik" im Juni 1981 attestiert hatte, stets "die demokratischen Prinzipien zu wahren und demokratische Prozesse zu achten"), um sie - nunmehr als Beweis demokratischen Engagements - zu demontieren, da eine sich über ihre ungebrochene Existenz vermittelte gesellschaftliche Polarisierung als zu risikoreich erwies. Der Machtwechsel von Marcos zu Corazon Aquino (25. Februar 1986) diente aus dieser Perspektive bereits als Rekonstruktion demokratischer Verhältnisse, was ein kritisches Würdigen der "People Power Revolution" erschwert.

Die weniger spektakulären Emanzipationsbestrebungen der Völker in der Region umfassend ins Blickfeld zu rücken und die vielfältigen Wurzeln/Entäußerungen ihres (auch bewaffneten) Protestes und Widerstandes bloßzulegen, fühlt sich das in Singapur bereits seit 1968 kontinuierlich recherchierende und publizierende Institute of Southeast Asian Studies (ISEAS) verpflichtet. Als Resultat einer im ISEAS intensiv geführten Debatte über die Notwendigkeit, sicherheitspolitisch und militärstrategisch relevante, globale Konzepte mit solchen spezifisch regionalen Zuschnitts zu verknüpfen und deren (vomehmlich von der Expertise südostasiatischer "scholars" bereicherter) Forschungsergebnisse "nicht nur den Regierungen und Militärs, sondem der akademischen Gemeinschaft, Massenmedien und, sofern möglich, auch der Geschäfts- und Handelswelt" zugänglich zu machen, entstand das u.a. von den Ford- und Rockefeller-Stiftungen finanziell unterstützte und dem ISEAS angegliederte Regional Strategic Studies Programme (RSSP). Dem von ISEASDirektor, Professor Kemial S. Sandhu, formulierten Anliegen, das RSSP möge dazu beitragen, "die Offentlichkeit über die Sicherheitsaspekte in der Region durch Diskussionen/ Seminare und Veröffentlichungen zu informieren" entsprach das Institut u.a. durch die Publizierung des Bandes "Armed Communist Movements in Southeast Asia". Ihm ordnet sich ergänzend die vorliegende Arbeit zu, die in sechs Kapiteln (einschließlich einer Einführung und Abschlußdiskussion) die Genese und Ausdrucksformen des bewaffneten Separatismus in Burma, Indonesien, den Philippinen und Thailand analysiert.

Da die Nationalstaaten Südostasiens ihren Untertanen nach wie vor hohe Tribute abverlangen und sich der Druck auf deren Sitten und Gebräuche im Falle von Opposition mit exponentiell wachsender Gewalt verstärkt, sei nicht, so hebt Ruth McVey (School of Oriental and African Studies/London) in ihrer Einführung hervor, das Warum bewaffneten Separatismus, sondern vielmehr die Frage entscheidend: Warum gibt es eigentlich nicht mehr davon? In der Tat: Eine auf den ersten Blick spannende wie provozierende Fragestellung. Analytisch präziser hätte eigentlich die Ausgangsfrage lauten müssen, was denn die konstitutiven Merkmale für den bis heute virulenten, aus politischen, wirtschaftlichen, religiösen und ethnischen Motiven gespeisten Widerstand sind und mittels welcher in- wie externer Faktoren es den herrschenden Eliten immer wieder gelingen konnte, ihn mit mehr oder minder großem Erfolg zu unterdrücken? Innerhalb eines solchen kategorialen Rahmens wären z.B. die historischen Formierungsprozesse des Etatismus, die landesspezifische 
Ausprägung seiner Stützen (Staatsbürokratie, Kompradoren etc.) und die theoretische Diskussion des Konstrukts "separatism" in ihrem Wechselspiel klarer bestimmbar gewesen. Anders formuliert: Zur umfassenden Erklärung des Phänomens "separatism" hätte sich als zentrale Frage geradezu aufgedrängt, was denn für den Prozeß der (vor)staatlichen Zentralisierung von Herrschaft verantwortlich war und wodurch sich der Staatsapparat und die ihn auszeichnenden Kräfte beispielsweise im (niemals kolonialisierten) Thailand und in den (jahrhundertelang kolonial durchdrungenen) Philippinen unterscheiden? Solche Fragen hätten in einem folgenden Schritt möglicherweise über die Charakterisierung des Umgangs mit ethnischen und anderen Minderheiten und den Grad ausländischer Intervention(sfähigkeit) hinaus die "separatistische" Dimension schärfer erschlossen, wie denn auch diese Dimension (von dem Sonderfall Burma abgesehen) sich in keinem der behandelten Fallbeispiele primär als solche äußerte. Last not least ist selbst die Terminologie bezeichnend:

"Bewaffneter Separatismus" setzt, obgleich die Studie des öfteren den weitergehenden Begriff "Sezession" insinuiert, per se die Wahrung zentralstaatlicher Instanzen voraus und neigt dazu, die oft legitimen sozialen und politischen Anliegen der "Separatisten" gegenüber den weitaus häufiger illegitimen Herrschaftsansprüchen regierender Eliten herabzusetzen. Vor diesem Hintergrund sind die Beiträge dieses Sammelbandes, der besser mit "State Responses of Selected Southeast Asian Countries vis-à-vis Armed Resistance" betitelt worden wäre, zu lesen und zu bewerten.

Trotzdem bietet der Band dem an der Region Interessierten einen informativen Einstieg in das - uns gewiß noch länger beschäftigende - Thema. Um es künftig tiefer und umfassender zu durchdringen, wäre es jedoch sinnvoll, nicht nur über die der Forschung unterworfenen "Subjekte" zu schreiben, sondern auch und gerade mit streitbaren Disput mit ihnen dauerhafte und den Emanzipationsbestrebungen der Betroffenen entsprechende Lösungen zu finden.

Rainer Werning

\section{J. Hermann Burgers / Hans Danelius}

The United Nations Convention against Torture. A Handbook on the Convention against Torture and Other Cruel, Inhuman or Degrading Treatment or Punishment Martinus Nijhoff Publishers, Dordrecht-Boston-London 1988, XII, 271 pp., $£ 44.95$

Die Antifolter-Konvention der Vereinten Nationen ist am 26.6.1987 in Kraft getreten. Das Werk von Burgers und Danelius erfüllt somit als Erläuterungswerk zu dem Abkommen einen wichtigen Zweck. Beide Autoren waren nicht nur bei der Ausarbeitung des Vertragswerks in den Gremien der Vereinten Nationen beteiligt, ihre Länder - und damit sie 